\title{
Convergence results of a matrix splitting algorithm for solving weakly nonlinear complementarity problems
}

\author{
Mei-Ju Luo*, Ya-Yi Wang and Hong-Ling Liu
}

"Correspondence:

meijuluolnu@126.com

School of Mathematics, Liaoning

University, Liaoning, 110036, China

\begin{abstract}
In this paper, we consider a class of weakly nonlinear complementarity problems (WNCP) with large sparse matrix. We present an accelerated modulus-based matrix splitting algorithm by reformulating the WNCP as implicit fixed point equations based on two splittings of the system matrixes. We show that, if the system matrix is a $P$-matrix, then under some mild conditions the sequence generated by the algorithm is convergent to the solution of WNCP.
\end{abstract}

MSC: $90 \mathrm{C} 33 ; 65 \mathrm{~F} 10 ; 65 \mathrm{~L} 10$

Keywords: weakly nonlinear complementarity problems; modulus-based; matrix splitting algorithm; convergence analysis

\section{Introduction}

Consider the following weakly nonlinear complementarity problems, which is to find $z \in$ $R^{n}$ such that

$$
z \geq 0, \quad A z+\Psi(z) \geq 0, \quad z^{T}(A z+\Psi(z))=0,
$$

abbreviated as WNCP, where $A=\left(a_{i j}\right) \in R^{n \times n}$ is a given large, sparse and real matrix, $\Psi(z)$ : $R^{n} \rightarrow R^{n}$ is a Lipschitz continuous nonlinear function, $z \geq 0$ means $z_{i} \geq 0, i=1,2, \ldots, n$, and $T$ always denotes the transpose of a vector.

As is well known, the classical nonlinear complementarity problems (NCP) are very important and fundamental topics in optimization theory and they have been developed into a well-established and fruitful principle. See [1-3] for details as regards the basic theories, effective algorithms and important applications of NCP. Problem (1) is a special case of NCP, but it extends from linear complementarity problems. When $\Psi(z)=q$ is a constant vector, problem (1) reduces to a linear complementarity problem. Recently, lots of researchers [4-8] have paid close attention to feasible and efficient methods for solving linear complementarity problems. Especially, by reformulating linear complementarity problems as an implicit fixed point equation, Van Bokhoven [9] proposed a modulus iteration method, which is defined as the solution of system of linear equations at each iteration. In 2010, Bai [10] presented a modulus-based matrix splitting iteration method and showed the convergence when the system matrix is an $H_{+}$-matrix. Consequently, Zhang

(c) 2016 Luo et al. This article is distributed under the terms of the Creative Commons Attribution 4.0 International License (http://creativecommons.org/licenses/by/4.0/), which permits unrestricted use, distribution, and reproduction in any medium, provided you give appropriate credit to the original author(s) and the source, provide a link to the Creative Commons license, and indicate if changes were made. 
[11] proposed two-step modulus-based matrix splitting iteration methods and considered the convergence theory when the system matrix is an $H_{+}$-matrix. Based on the above work, for solving problem (1), in [12] Sun and Zeng proposed a modified semismooth Newton method with $A$ being an $M$-matrix and $\Psi(z)$ being a continuously differentiable monotone diagonal function on $R^{n}$.

In this paper, for satisfying the requirements of the application, more details can be found in [13-16], we present an accelerated modulus-based matrix splitting algorithm for dealing with WNCP. The organization of this paper is as follows: Some necessary notations and definitions are introduced in Section 2. In Section 3, we establish a class of accelerated modulus-based matrix splitting iteration algorithms. In Section 4, the convergence conditions are considered.

\section{Preliminaries}

Some necessary notations, definitions and lemmas used in the sequel discussions are introduced in this section. For $B \in R^{n \times n}$, we write $B^{-1}, B^{T}, \rho(B)$ to denote the inverse, the transpose, the spectral radius of the matrix $B$, respectively. For $x \in R^{n}$, we write $\|x\|,|x|$ to denote the norm of the vector $x,|x|=\left(\left|x_{1}\right|, \ldots,\left|x_{n}\right|\right)$, respectively. $\|A\|$ denotes any norm of matrix $A$. Especially, we use $\|\cdot\|_{2}$ to denote a spectral norm. $\lambda \in \lambda(A)$ denotes the eigenvalue of matrix $A$ where $\lambda(A)$ is the set of all eigenvalues of matrix $A$.

Definition 1 [17] If for any $x:=\left(x_{1}, x_{2}, \ldots, x_{n}\right) \neq 0$, there exists an index $k$, such that $x_{k}(A x)_{k}=x_{k}\left(a_{k 1} x_{1}+\cdots+a_{k n} x_{n}\right)>0$, we call that matrix $A$ is a P-matrix.

Definition 2 For the function $f(x): R^{n} \rightarrow R^{n}$, iffor any $x, y \in R^{n}$, there exists a constant $L$ such that

$$
\|f(x)-f(y)\| \leq L\|x-y\|
$$

then we call $f$ a Lipschitz continuous function on $R^{n}$, and L is called a Lipschitz constant.

Lemma 3 [18] Let $A=\left(a_{i j}\right) \in R^{n \times n}$ be a P-matrix, for any nonnegative diagonal matrix $\Omega$, the matrix $A+\Omega$ is nonsingular.

\section{Algorithm}

Theorem 4 Let $M_{1}-N_{1}=M_{2}-N_{2}=A$ be two splittings of the matrix $A \in R^{n \times n}$ and $\Omega, \Gamma$ be $n \times n$ positive diagonal matrices, $\Omega_{1}, \Omega_{2}$ be $n \times n$ nonnegative diagonal matrices such that $\Omega=\Omega_{1}+\Omega_{2}$, then the following statements hold.

(i) If $z$ is a solution of problem (1), then $x=\frac{1}{2}\left(\Gamma^{-1} z-\Omega^{-1}(A z+\Psi(z))\right)$ satisfies the implicit fixed point equation

$$
\left(M_{1} \Gamma+\Omega_{1}\right) x=\left(N_{1} \Gamma-\Omega_{2}\right) x+\left(\Omega-M_{2} \Gamma\right)|x|+N_{2} \Gamma|x|-\Psi(\Gamma(|x|+x)) .
$$

(ii) If $x$ satisfies the implicit fixed point equation (2), then

$$
z=\Gamma(|x|+x)
$$

is a solution of problem (1). 
Proof First we prove part (i). Since $z$ is a solution of problem (1), we have $z \geq 0$. So there exists $x \in R^{n}$ such that

$$
z=\Gamma(|x|+x) .
$$

Define another nonnegative vector,

$$
v=\Omega(|x|-x)
$$

It is easy to see that $v \geq 0, z^{T} v=0$, and $v=A z+\Psi(z)$ if and only if

$$
\Omega(|x|-x)=A \Gamma(|x|+x)+\Psi(\Gamma(|x|+x)) .
$$

Replace $A$ with $M_{1}-N_{1}$, we have

$$
\left(M_{1} \Gamma+\Omega\right) x=N_{1} \Gamma x+\left(\Omega-\left(M_{1}-N_{1}\right) \Gamma\right)|x|-\Psi(\Gamma(|x|+x)) .
$$

Taking $M_{1}-N_{1}=M_{2}-N_{2}$ and $\Omega=\Omega_{1}+\Omega_{2}$ into account, it follows that

$$
\left(M_{1} \Gamma+\Omega_{1}\right) x=\left(N_{1} \Gamma-\Omega_{2}\right) x+\left(\Omega-M_{2} \Gamma\right)|x|+N_{2} \Gamma|x|-\Psi(\Gamma(|x|+x)) .
$$

This shows that equation (2) holds.

We now turn to prove part (ii). By some simple calculations, the implicit fixed point equation (2) is equivalent to

$$
A \Gamma(|x|+x)+\Psi(\Gamma(|x|+x))=\Omega(|x|-x)
$$

Set $z=\Gamma(|x|+x)$ and $v=\Omega(|x|-x)$. Evidently, it yields $z \geq 0, v \geq 0, z^{T} v=0$, and $v=$ $A z+\Psi(z)$, which means that $z$ is a solution of problem (1). This completes the proof.

Note that the implicit fixed point equation (2) includes many parameters that are quite complicated to be determined in a computation. For solving this problem, we will give the simple formulation as follows. Subsequently, a matrix splitting iteration algorithm will be given for solving WNCP. Let $\Omega_{1}=\Omega, \Omega_{2}=0, \Gamma=\frac{1}{\gamma} I$, where $\gamma>0$ is a real number, then the implicit fixed point equation reduces to

$$
\left(M_{1}+\gamma \Omega\right) x=N_{1} x+\left(\gamma \Omega-M_{2}\right)|x|+N_{2}|x|-\gamma \Psi\left(\frac{(|x|+x)}{\gamma}\right) .
$$

In fact, $\gamma \Omega$ in the above equation denotes a positive diagonal parameter matrix, which can be replaced by $\Omega$ for simplicity. That is, the above equation is essentially equivalent to

$$
\left(M_{1}+\Omega\right) x=N_{1} x+\left(\Omega-M_{2}\right)|x|+N_{2}|x|-\gamma \Psi\left(\frac{(|x|+x)}{\gamma}\right) .
$$

In the rest of the paper, we will use the fixed point equation above to give the algorithm and convergence analysis for solving WNCP. 


\section{Algorithm 5}

Step 1. Choose two splittings of the matrix $A \in R^{n \times n}$ satisfying $A=M_{1}-N_{1}=M_{2}-N_{2}$.

Step 2. Set $k=0$. Give an initial vector $x^{0} \in R^{n}$, compute $z^{0}=\frac{1}{\gamma}\left(\left|x^{0}\right|+x^{0}\right)$.

Step 3. Choose $x^{k+1}$ such that

$$
\left(M_{1}+\Omega\right) x^{k+1}=N_{1} x^{k}+\left(\Omega-M_{2}\right)\left|x^{k}\right|+N_{2}\left|x^{k+1}\right|-\gamma \Psi\left(z^{k}\right),
$$

and set

$$
z^{k+1}=\frac{1}{\gamma}\left(\left|x^{k+1}\right|+x^{k+1}\right), \quad k=k+1
$$

Here, $\Omega, \Gamma \in R^{n \times n}$ are positive diagonal matrices and $\gamma$ is a positive constant.

Step 4. If the sequence $\left\{z^{k}\right\}_{0}^{+\infty}$ is convergent, stop. Otherwise, go to Step 3.

\section{Convergence theorems}

In this section, we will consider the conditions that ensure the convergence of $\left\{z^{k}\right\}_{0}^{+\infty}$ obtained by Algorithm 5 .

Theorem 6 Let $A \in R^{n \times n}$ be a P-matrix, $M_{1}-N_{1}=M_{2}-N_{2}=A$ be two splittings of the matrix $A$ with $M_{1} \in R^{n \times n}$ being a P-matrix. Assume that $\Omega \in R^{n \times n}$ is a positive diagonal matrix, $\gamma$ is a positive constant and $\Psi(z): R^{n} \rightarrow R^{n}$ is a Lipschitz continuous function with the Lipschitz constant L. Set

$$
\rho(\Omega)=2 g(\Omega)+2 q(\Omega)+f(\Omega)
$$

where $g(\Omega)=\left\|\left(M_{1}+\Omega\right)^{-1} N_{2}\right\|, q(\Omega)=\left\|\left(M_{1}+\Omega\right)^{-1} N_{1}\right\|+L\left\|\left(M_{1}+\Omega\right)^{-1}\right\|, f(\Omega)=\|\left(M_{1}+\right.$ $\Omega)^{-1}\left(\Omega-M_{1}\right) \|$. If the matrix $\Omega$ satisfies $\rho(\Omega)<1$, then for any initial vector $x^{0} \in R^{n}$, the iteration sequence $\left\{z^{k}\right\}_{k=0}^{+\infty}$ generated by Algorithm 5 converges to a solution $z^{*} \in R_{+}^{n}$ of prob$\operatorname{lem}(1)$.

Proof Suppose that $z^{*} \in R_{+}^{n}$ is a solution of problem (1). Note that $\Gamma=\frac{1}{\gamma} I$, by Theorem 4 and (3), we see that $x^{*}=\frac{1}{2}\left(\gamma z^{*}-\Omega^{-1}\left(A\left(z^{*}\right)+\Psi\left(z^{*}\right)\right)\right)$ is a solution of the equation

$$
\left(M_{1}+\Omega\right) x^{*}=N_{1} x^{*}+\left(\Omega-M_{2}\right)\left|x^{*}\right|+N_{2}\left|x^{*}\right|-\gamma \Psi\left(z^{*}\right)
$$

with $z^{*}=\frac{1}{\gamma}\left(\left|x^{*}\right|+x^{*}\right)$. Let us consider (4) minus (5); we have

$$
\begin{aligned}
& \left(M_{1}+\Omega\right)\left(x^{k+1}-x^{*}\right) \\
= & N_{1}\left(x^{k}-x^{*}\right)+\left(\Omega-M_{2}\right)\left(\left|x^{k}\right|-\left|x^{*}\right|\right) \\
& +N_{2}\left(\left|x^{k+1}\right|-\left|x^{*}\right|\right)-\gamma\left(\Psi\left(z^{k}\right)-\Psi\left(z^{*}\right)\right) .
\end{aligned}
$$

Note that $M_{1}-N_{1}=M_{2}-N_{2}$, we have

$$
\begin{aligned}
& \left(\Omega-M_{2}\right)\left(\left|x^{k}\right|-\left|x^{*}\right|\right) \\
= & \left(\Omega-M_{2}+N_{2}-N_{2}\right)\left(\left|x^{k}\right|-\left|x^{*}\right|\right)
\end{aligned}
$$




$$
\begin{aligned}
& =\left(\Omega-M_{1}+N_{1}-N_{2}\right)\left(\left|x^{k}\right|-\left|x^{*}\right|\right) \\
& =\left(\Omega-M_{1}\right)\left(\left|x^{k}\right|-\left|x^{*}\right|\right)+N_{1}\left(\left|x^{k}\right|-\left|x^{*}\right|\right)-N_{2}\left(\left|x^{k}\right|-\left|x^{*}\right|\right) .
\end{aligned}
$$

Since $M_{1}$ is a $P$-matrix and $\Omega$ is a positive diagonal matrix, it follows from Lemma 3 that $M_{1}+\Omega$ is a nonsingular matrix. Hence, by (5) and (6), we have

$$
\begin{aligned}
& x^{k+1}-x^{*} \\
= & \left(M_{1}+\Omega\right)^{-1} N_{1}\left(x^{k}-x^{*}\right)+\left(M_{1}+\Omega\right)^{-1}\left(\Omega-M_{1}\right)\left(\left|x^{k}\right|-\left|x^{*}\right|\right) \\
& +\left(M_{1}+\Omega\right)^{-1} N_{1}\left(\left|x^{k}\right|-\left|x^{*}\right|\right)-\left(M_{1}+\Omega\right)^{-1} N_{2}\left(\left|x^{k}\right|-\left|x^{*}\right|\right) \\
& +\left(M_{1}+\Omega\right)^{-1} N_{2}\left(\left|x^{k+1}\right|-\left|x^{*}\right|\right)-\left(M_{1}+\Omega\right)^{-1} \gamma\left(\Psi\left(z^{k}\right)-\Psi\left(z^{*}\right)\right) .
\end{aligned}
$$

Taking the facts

$$
\left\|z^{k}-z^{*}\right\|=\left\|\frac{\left|x^{k}\right|+x^{k}}{\gamma}-\frac{\left|x^{*}\right|+x^{*}}{\gamma}\right\| \leq \frac{2}{\gamma}\left\|x^{k}-x^{*}\right\|,
$$

$\Psi(z)$ is a Lipschitz continuous function, and (8) into account, we have

$$
\left\|\Psi\left(z^{k}\right)-\Psi\left(z^{*}\right)\right\| \leq L\left\|z^{k}-z^{*}\right\| \leq \frac{2 L}{\gamma}\left\|x^{k}-x^{*}\right\| .
$$

Thereby, we derive from (7) and (9) that

$$
\begin{aligned}
& \left(1-\left\|\left(M_{1}+\Omega\right)^{-1} N_{2}\right\|\right)\left\|x^{k+1}-x^{*}\right\| \\
\leq & \left(2\left(\left\|\left(M_{1}+\Omega\right)^{-1} N_{1}\right\|+L\left\|\left(M_{1}+\Omega\right)^{-1}\right\|\right)+\left\|\left(M_{1}+\Omega\right)^{-1}\left(\Omega-M_{1}\right)\right\|\right. \\
& \left.+\left\|\left(M_{1}+\Omega\right)^{-1} N_{2}\right\|\right)\left\|x^{k}-x^{*}\right\|,
\end{aligned}
$$

which is equivalent to

$$
\left\|x^{k+1}-x^{*}\right\| \leq \frac{2 q(\Omega)+f(\Omega)+g(\Omega)}{1-g(\Omega)}\left\|x^{k}-x^{*}\right\|
$$

with $g(\Omega)<1$. The condition

$$
\frac{2 q(\Omega)+f(\Omega)+g(\Omega)}{1-g(\Omega)}<1
$$

with $g(\Omega)<1$, which is equivalent to $\rho(\Omega)=2 g(\Omega)+2 q(\Omega)+f(\Omega)<1$, ensures that the limit $\lim _{k \rightarrow+\infty} x^{k}=x^{*}$ holds. These results complete the proof.

Theorem 7 Let $A \in R^{n \times n}$ be a P-matrix, $M_{1}-N_{1}=M_{2}-N_{2}=A$ be two splittings of the matrix $A$ with $M_{1} \in R^{n \times n}$ being a symmetric P-matrix. Suppose that $\Omega=\omega I \in R^{n \times n}$ is a positive scalar matrix and $\omega$ is a positive constant. $\Psi(z): R^{n} \rightarrow R^{n}$ is a Lipschitz continuous function with the Lipschitz constant $L . \lambda_{\max }$ and $\lambda_{\min }$ to denote the largest and smallest eigenvalue of the matrix $M_{1}$, respectively. Let $\tau_{1}=\left\|M_{1}^{-1} N_{1}\right\|_{2}$ and $\tau_{2}=\left\|M_{1}^{-1} N_{2}\right\|_{2}$ satisfy $\tau_{1}+\tau_{2}<1$. If $\lambda_{\min }>L$, the choices of the parameters $\omega, M_{1}, N_{1}, M_{2}, N_{2}$ satisfy either of the 
following conditions, then the iteration sequence $\left\{z_{k}\right\}_{k}^{+\infty} \subset R_{+}^{n}$ generated by Algorithm 5 converges to the unique solution $z^{*} \in R_{+}^{n}$ of WNCP for any initial vector $x^{0} \in R^{n}$.

(i) When $0<\tau_{1}+\tau_{2}<\frac{\lambda_{\min }-L}{\lambda_{\max }}$,

$$
\omega=\sqrt{\lambda_{\max } \lambda_{\min }}
$$

(ii) When $\frac{\lambda_{\min }-L}{\lambda_{\max }}<\tau_{1}+\tau_{2}<\frac{\lambda_{\min }-L}{\sqrt{\lambda_{\max } \lambda_{\min }}}$,

$$
\sqrt{\lambda_{\max } \lambda_{\min }} \leq \omega<\frac{\left[1-\left(\tau_{1}+\tau_{2}\right)\right] \lambda_{\max } \lambda_{\min }-L \lambda_{\max }}{\left(\tau_{1}+\tau_{2}\right) \lambda_{\max }+L-\lambda_{\min }} .
$$

(iii) When $\tau_{1}+\tau_{2}=\frac{\lambda_{\min }-L}{\lambda_{\max }}$,

$$
\omega \geq \sqrt{\lambda_{\max } \lambda_{\min }}
$$

Proof We first give some formulations, which will be used in the proof. Since $M$ is a symmetric $P$-matrix and $\tau_{1}+\tau_{2}<1$, by the definition of the spectral norm, we have

$$
\begin{aligned}
\left\|\left(M_{1}+\Omega\right)^{-1} N_{1}\right\|_{2} & =\left\|\left(M_{1}+\omega I\right)^{-1} M_{1} M_{1}^{-1} N_{1}\right\|_{2} \\
& \leq\left\|\left(M_{1}+\omega I\right)^{-1} M_{1}\right\|_{2}\left\|M_{1}^{-1} N_{1}\right\|_{2} \\
& =\max _{\lambda \in \lambda\left(M_{1}\right)} \frac{\lambda \tau_{1}}{\omega+\lambda} \\
& =\frac{\lambda_{\max } \tau_{1}}{\omega+\lambda_{\max }} .
\end{aligned}
$$

Similarly, we have

$$
\begin{aligned}
\left\|\left(M_{1}+\Omega\right)^{-1} N_{2}\right\|_{2} & =\left\|\left(M_{1}+\omega I\right)^{-1} M_{1} M_{1}^{-1} N_{1}\right\|_{2} \\
& \leq \frac{\lambda_{\max } \tau_{2}}{\omega+\lambda_{\max }}
\end{aligned}
$$

and

$$
\begin{aligned}
\left\|\left(M_{1}+\Omega\right)^{-1}\right\|_{2} & =\left\|\left(M_{1}+\omega I\right)^{-1}\right\|_{2} \\
& =\max _{\lambda \in \lambda\left(M_{1}\right)} \frac{1}{\omega+\lambda} \\
& =\frac{1}{\omega+\lambda_{\text {min }}} .
\end{aligned}
$$

In addition, from a simple calculating process, we have

$$
\begin{aligned}
\left\|\left(M_{1}+\Omega\right)^{-1}\left(\Omega-M_{1}\right)\right\|_{2} & =\left\|\left(M_{1}+\omega I\right)^{-1}\left(\omega I-M_{1}\right)\right\|_{2} \\
& =\max _{\lambda \in \lambda\left(M_{1}\right)} \frac{|\omega-\lambda|}{\omega+\lambda}=\max \left\{\frac{\left|\omega-\lambda_{\max }\right|}{\omega+\lambda_{\max }}, \frac{\left|\omega-\lambda_{\min }\right|}{\omega+\lambda_{\min }}\right\} \\
& = \begin{cases}\frac{\lambda_{\max }-\omega}{\lambda_{\max }+\omega}, & \omega \leq \sqrt{\lambda_{\max } \lambda_{\min }}, \\
\frac{\omega-\lambda_{\min }}{\omega+\lambda_{\min }}, & \omega \geq \sqrt{\lambda_{\max } \lambda_{\min } .}\end{cases}
\end{aligned}
$$


As follows from (10)-(13), we have

$$
\begin{aligned}
\rho(\Omega)= & 2 g(\Omega)+2 q(\Omega)+f(\Omega) \\
= & 2\left\|\left(M_{1}+\Omega\right)^{-1} N_{2}\right\|_{2}+2\left(\left\|\left(M_{1}+\Omega\right)^{-1} N_{1}\right\|_{2}\right. \\
& \left.+L\left\|\left(M_{1}+\Omega\right)^{-1}\right\|_{2}\right)+\left\|\left(M_{1}+\Omega\right)^{-1}\left(\Omega-M_{1}\right)\right\|_{2} \\
= & 2 \frac{\lambda_{\max } \tau_{2}}{\omega+\lambda_{\max }}+2\left(\frac{\lambda_{\max } \tau_{1}}{\omega+\lambda_{\max }}+\frac{L}{\omega+\lambda_{\min }}\right)+ \begin{cases}\frac{\lambda_{\max }-\omega}{\omega+\lambda_{\max }}, & \omega \leq \sqrt{\lambda_{\max } \lambda_{\min }}, \\
\frac{\omega-\lambda_{\min }}{\omega+\lambda_{\min }}, & \omega \geq \sqrt{\lambda_{\max } \lambda_{\min } .}\end{cases}
\end{aligned}
$$

We then consider two cases.

(a) When $\omega \leq \sqrt{\lambda_{\max } \lambda_{\min }}$, by a simple calculation on (14), we see that $\omega, \tau_{1}$, and $\tau_{2}$ satisfy $\rho(\Omega)<1$, which is equivalent to

$$
\omega^{2}-\left(\left(\tau_{1}+\tau_{2}\right) \lambda_{\max }+L-\lambda_{\min }\right) \omega-\left(\left(\tau_{1}+\tau_{2}\right) \lambda_{\max } \lambda_{\min }+L \lambda_{\max }\right)>0 .
$$

Note that $\omega>0$ and $\omega \leq \sqrt{\lambda_{\max } \lambda_{\min }}$, then the solution of the above inequality is

$$
\theta\left(\tau_{1}, \tau_{2}\right)<\omega \leq \sqrt{\lambda_{\max } \lambda_{\min }}
$$

where

$$
\begin{aligned}
\theta\left(\tau_{1}, \tau_{2}\right) \\
=\frac{\sqrt{\left(\left(\tau_{1}+\tau_{2}\right) \lambda_{\max }+L-\lambda_{\min }\right)^{2}+4\left(\tau_{1}+\tau_{2}\right) \lambda_{\max } \lambda_{\min }+L \lambda_{\max }}}{2} \\
+\frac{\left(\tau_{1}+\tau_{2}\right) \lambda_{\max }+L-\lambda_{\min }}{2} .
\end{aligned}
$$

Certainly, we have

$$
\theta\left(\tau_{1}, \tau_{2}\right)<\sqrt{\lambda_{\max } \lambda_{\min }}
$$

Since $\lambda_{\min }>L$, by the definitions of $\tau_{1}, \tau_{2}$ and solving (15), we get

$$
0<\tau_{1}+\tau_{2}<\frac{\lambda_{\min }-L}{\sqrt{\lambda_{\max } \lambda_{\min }}} .
$$

(b) When $\omega \geq \sqrt{\lambda_{\max } \lambda_{\min }}$, in a same way as (a), $\omega, \tau_{1}$, and $\tau_{2}$ satisfying $\rho(\Omega)<1$, which is equivalent to

$$
\left[\left(\tau_{1}+\tau_{2}\right) \lambda_{\max }+L-\lambda_{\min }\right] \omega+\left(\tau_{1}+\tau_{2}-1\right) \lambda_{\max } \lambda_{\min }+L \lambda_{\max }<0
$$

$$
\begin{aligned}
& \text { If }\left(\tau_{1}+\tau_{2}\right) \lambda_{\max }+L-\lambda_{\min }>0 \text {, that is, } \tau_{1}+\tau_{2}>\frac{\lambda_{\min }-L}{\lambda_{\max }} \text {, then } \\
& \omega<\frac{\left[1-\left(\tau_{1}+\tau_{2}\right)\right] \lambda_{\max } \lambda_{\min }-L \lambda_{\max }}{\left(\tau_{1}+\tau_{2}\right) \lambda_{\max }+L-\lambda_{\min }} .
\end{aligned}
$$


Combined with $\omega \geq \sqrt{\lambda_{\max } \lambda_{\min }}$, we get

$$
\sqrt{\lambda_{\max } \lambda_{\min }} \leq \omega<\frac{\left[1-\left(\tau_{1}+\tau_{2}\right)\right] \lambda_{\max } \lambda_{\min }-L \lambda_{\max }}{\left(\tau_{1}+\tau_{2}\right) \lambda_{\max }+L-\lambda_{\min }} .
$$

Naturally, we have

$$
\sqrt{\lambda_{\max } \lambda_{\min }}<\frac{\left[1-\left(\tau_{1}+\tau_{2}\right)\right] \lambda_{\max } \lambda_{\min }-L \lambda_{\max }}{\left(\tau_{1}+\tau_{2}\right) \lambda_{\max }+L-\lambda_{\min }} .
$$

That is,

$$
\tau_{1}+\tau_{2}<\frac{\lambda_{\min }-L}{\sqrt{\lambda_{\max } \lambda_{\min }}} .
$$

This, together with $\tau_{1}+\tau_{2}>\frac{\lambda_{\min }-L}{\lambda_{\max }}$, shows that we have

$$
\begin{aligned}
& \frac{\lambda_{\min }-L}{\lambda_{\max }}<\tau_{1}+\tau_{2}<\frac{\lambda_{\min }-L}{\sqrt{\lambda_{\max } \lambda_{\min }}} . \\
& \text { If }\left(\tau_{1}+\tau_{2}\right) \lambda_{\max }+L-\lambda_{\min } \leq 0 \text {, that is, } \tau_{1}+\tau_{2} \leq \frac{\lambda_{\min }-L}{\lambda_{\max }} \text {, then for any } \omega>0 \text { (16) holds. So } \\
& \omega \geq \sqrt{\lambda_{\max } \lambda_{\min }} .
\end{aligned}
$$

Hence, from (a) and (b), we see that when $0<\tau_{1}+\tau_{2}<\frac{\lambda_{\min }-L}{\lambda_{\max }}, \omega=\sqrt{\lambda_{\max } \lambda_{\min }}$; when $\frac{\lambda_{\min }-L}{\lambda_{\max }}<\tau_{1}+\tau_{2}<\frac{\lambda_{\min }-L}{\sqrt{\lambda_{\max } \lambda_{\min }}}, \sqrt{\lambda_{\max } \lambda_{\min }} \leq \omega<\frac{\left[1-\left(\tau_{1}+\tau_{2}\right)\right] \lambda_{\max } \lambda_{\min }-L \lambda_{\max }}{\left(\tau_{1}+\tau_{2}\right) \lambda_{\max }+L-\lambda_{\min }}$; when $\tau_{1}+\tau_{2}=\frac{\lambda_{\min }-L}{\lambda_{\max }}$, $\omega \geq \sqrt{\lambda_{\max } \lambda_{\min }}$. The proof is completed.

\section{Results and discussion}

This study focused on the weakly nonlinear complementarity problems with a large sparse matrix. We proposed an algorithm that is not only computationally more convenient to use but also faster than the modulus-based matrix splitting iteration methods and the convergence conditions are presented when the system matrix is a $P$-matrix.

Some scholars had already stressed the accelerated modulus-based matrix splitting iteration methods for linear complementarity problems and pointed out that the system matrix is either a positive definite matrix or an $H_{+}$-matrix. However, we suggest that the system matrix is a $P$-matrix, this is more adaptable but also a limitation. Notwithstanding its limitation, this study does suggest that WNCP can be solved faster.

\section{Conclusions}

In this paper, by reformulating the complementarity problem (1) as an implicit fixed point equation based on splittings of the system matrix $A$, we establish an accelerated modulusbased matrix splitting iteration algorithm and show the convergence analysis when the involved matrix of the WNCP is a $P$-matrix.

Competing interests

The authors declare that they have no competing interests. 


\section{Acknowledgements}

This work was supported in part by NSFC Grant NO. 11501275 and Scientific Research Fund of Liaoning Provincial Education Department NO. L2015199.

Received: 13 April 2016 Accepted: 28 July 2016 Published online: 22 August 2016

\section{References}

1. Lin, GH, Fukushima, M: New reformulations for stochastic nonlinear complementarity problems. Optim. Methods Softw. 21, 551-564 (2006)

2. Tseng, P: Growth behavior of a class of merit functions for the nonlinear complementarity problem. J. Optim. Theory Appl. 89, 17-37 (1996)

3. Zhang, C, Chen, XJ: Stochastic nonlinear complementarity problem and applications to traffic equilibrium under uncertainty. J. Optim. Theory Appl. 137, 277-295 (2008)

4. Chen, XJ, Zhang, C, Fukushima, M: Robust solution of monotone stochastic linear complementarity problems. Math. Program. 117, 51-80 (2009)

5. Fang, $\mathrm{H}$, Chen, $\mathrm{X}$, Fukushima, M: Stochastic $R_{0}$ matrix linear complementarity problems. SIAM J. Optim. 18, 482-506 (2007)

6. Fukushima, M: Equivalent differentiable optimization problems and descent methods for asymmetric variational inequality problems. Math. Program. 137, 99-110 (1992)

7. Zhang, C, Chen, XJ: Smoothing projected gradient method and its application to stochastic linear complementarity problems. SIAM J. Optim. 20, 627-649 (2009)

8. Zhou, G, Caccetta, L: Feasible semismooth Newton method for a class of stochastic linear complementarity problems. J. Optim. Theory Appl. 139, 379-392 (2008)

9. Van Bokhoven, WM: A class of linear complementarity problems is solvable in polynomial time. Technical report, Department of Electrical Engineering, Technical University, Eindhoven (1980)

10. Bai, Z: Modulus-based matrix splitting iteration methods for linear complementarity problems. Numer. Linear Algebra Appl. 6, 917-933 (2010)

11. Zhang, L: Two-step modulus based matrix splitting iteration for linear complementarity problems. Numer. Algorithms 57, 83-99 (2011)

12. Sun, Z, Zeng, J: A monotone semismooth Newton type method for a class of complementarity problems. J. Comput. Appl. Math. 235, 1261-1274 (2011)

13. Chung, K: Equivalent Differentiable Optimization Problems and Descent Methods for Asymmetric Variational Inequality Problems. Academic Press, New York (1992)

14. Fukushima, M: Merit functions for variational inequality and complementarity problems. In: Nonlinear Optimization and Applications, vol. 137, pp. 155-170 (1996)

15. Lin, GH, Chen, XJ, Fukushima, M: New restricted NCP function and their applications to stochastic NCP and stochastic MPEC. Optimization 56, 641-753 (2007)

16. Ling, C, Qi, L, Zhou, G: The $S C^{1}$ property of an expected residual function arising from stochastic complementarity problems. Oper. Res. Lett. 36, 456-460 (2008)

17. Schafer, U: A linear complementarity problem with a P-matrix. SIAM Rev. 46, 189-201 (2004)

18. Berman, A, Plemmons, R: Nonnegative Matrices in the Mathematical Sciences. Academic Press, New York (1979)

\section{Submit your manuscript to a SpringerOpen ${ }^{\circ}$ journal and benefit from:}

- Convenient online submission

Rigorous peer review

- Immediate publication on acceptance

- Open access: articles freely available online

- High visibility within the field

- Retaining the copyright to your article 